\title{
How do microsporidia invade cells?
}

\section{REVIEW ARTICLE}

\author{
Caspar Franzen
}

Department of Internal Medicine I, University of Regensburg, 93042 Regensburg, Germany

Key words: Microsporidia, Encephalitozoon, cell invasion, parasitophorous vacuole, phagocytosis, endocytosis

\begin{abstract}
Microsporidia are obligate intracellular eukaryotic parasites that utilize a unique mechanism to infect host cells. One of the main characteristics of all microsporidia is that they produce spores containing an extrusion apparatus that consists of a coiled polar tube ending in an anchoring disc at the apical part of the spore. With appropriate conditions inside a suitable host, the polar tube is discharged through the thin anterior end of the spore, thereby penetrating a new host cell for inoculating the infective sporoplasm into the new host cell. This method of invading new host cells is one of the most sophisticated infection mechanisms in biology and ensures that the microsporidia enter the host cell unrecognized and protected from the host defence reactions. Recent studies have shown that microsporidia gain access to host cells by phagocytosis as well. However, after phagocytosis, the special infection mechanism of the microsporidia is used to escape from the maturing phagosomes and to infect the cytoplasm of the cells. Gaining access to cells by endocytosis, and escaping destruction in the phago-/endo-/lysosome by egressing quickly from the phagocytic vacuole to multiply outside the lysosome, is a common phenomenon in biology that has been evolved several times during evolution. How this is put into execution by the microsporidia is an inimitable principle by which an obligate intracellular organism has managed this problem. The extrusion apparatus of the microsporidia has obviously ensured the success of this phylum during evolution, resulting in a group of obligate intracellular organisms, capable of infecting almost any type of host and cell.
\end{abstract}

\section{INTRODUCTION}

Microsporidia are an unusual group of obligate intracellular parasites that have no active stages outside their host cells. They can survive only by living inside other cells and their method of invading new host cells is really astounding. One of the main characteristics of all microsporidia is that they produce spores with a unique ultrastructure (Fig. 1). These spores contain an extrusion apparatus that consists of a coiled polar tube ending in an anchoring disc at the apical part of the spore (Vávra and Larsson 1999). With appropriate conditions inside a suitable host, the polar tube is discharged through the thin anterior end of the spore, thereby penetrating a new host cell for inoculating the infective sporoplasm through the hollow tube into the new host cell. Inside the host cells, the sporoplasms that are released from the spores become meronts that develop into sporonts which are characterized by the appearance of a dense surface coat. Sporonts divide into sporoblasts that will finally develop to mature spores. Since there are over 1,000 species of microsporidia, the life cycles are quite variable: simple life cycles are completed in a single host, whereas complex cycles require different hosts with involvement of more than one generation of the parasite that produce different kind of spores with different morphologies (Cali and Takvorian 1999). Infection by microsporidia has been described for different kinds of cells and microsporidia of the genus Encephalitozoon infect a wide range of human cell types

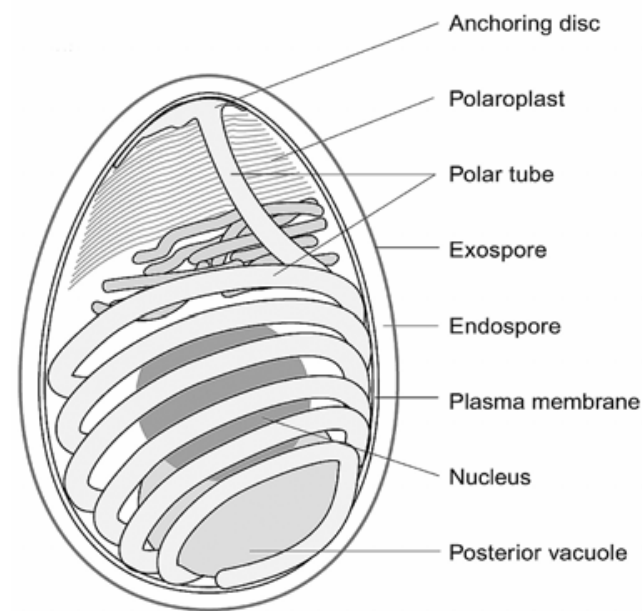

Fig. 1. Diagram of a microsporidian spore. The spore wall is composed of three layers: an electron-dense exospore, a thick electron-lucent endospore, and a plasma membrane. The unique extrusion apparatus inside the spore consists of the coiled polar tube (the number of coils depends on the particular species and varies from a few to 30 or more) that is ending at the apical part of the spore in an anchoring disk. Furthermore inside the spore there is the polaroplast, ribosomes, the nucleus, and the posterior vacuole. (Reprinted with permission from Franzen 2004.)

This paper was presented at the NATO Advanced Research Workshop "Emergent Pathogens in the 21st Century: First United Workshop on Microsporidia from Invertebrate and Vertebrate Hosts", held in České Budějovice, Czech Republic, July 12-15, 2004. 


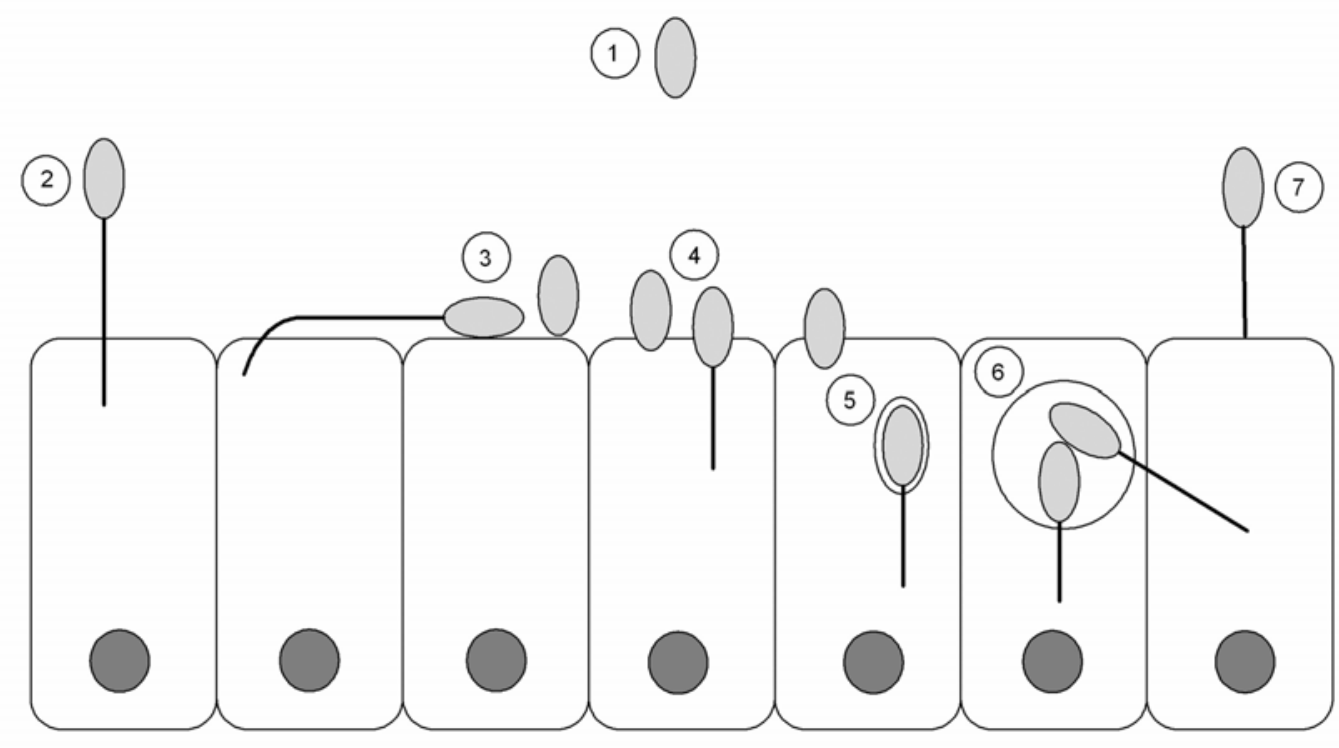

Fig. 2. Hypothetical concepts of cell invasion of microsporidia. 1. Free microsporidian spore. 2. Piercing of the host cell membrane by the extruded polar tube and injection of the sporoplasm into the host cell. 3. Interaction between the microsporidian spore and the cellular membrane, extrusion of the polar tube, and induced phagocytosis of the sporoplasm within a host cell membrane-derived vacuole. 4. Piercing of the host cell membrane by the extruded polar tube and injection of the sporoplasm into the host cell after interaction between the microsporidian spore and the cellular membrane and initiation of endocytosis of the spore. 5. Phagocytosis of the spore and escape of the sporoplasm from the maturing phago-/endo-/lysosome by polar tube discharge. 6. Secondary infection, starting from the parasitophorous vacuole (in the case of Encephalitozoon spp.) or from the cell cytoplasm, of the same or of an adjacent cell through the polar tube. 7. Phagocytosis of the previously extruded polar tube. (Modified after Vivarès and Méténier 2001.)

including epithelial cells, endothelial cells, kidney tubule cells, and macrophages (Visvesvara 2002). The method of invading new host cells used by microsporidia is one of the most sophisticated infection mechanisms in biology and ensures that the microsporidia enter the host cell unrecognized and protected from the host defence reactions. Although the unique extrusion apparatus of the microsporidia and the way it is used to infect new host cells has been described in detail (Weidner 1972, Keohane and Weiss 1999, Cali et al. 2002), several questions still remain and will be discussed below.

\section{Cell invasion}

The polar tube is the most intriguing structure of the microsporidia and has attracted the attention of parasitologists for a long time. It has been over 100 years since the microsporidian polar tube and the triggering of its discharge has been described (Thélohan 1892, 1894), but how the polar tube penetrates the host cell membrane is still unclear. The polar tube has a very narrow diameter $(0.1$ to $0.2 \mu \mathrm{m})$ and is emitted from the spore with considerable force. Initially, it was thought that it pierces the plasma membrane of the new host cell so that the polar tube is used like a hypodermic needle to inject the sporoplasm into the host cytoplasm. Recent studies using Encephalitozoon spp. have suggested that the polar tube may enter new host cells by a phagocytic process. The possible development of such a penetration process can be seen on scanning electron micrographs of Encephalitozoon spp. including the invagination tunnel in which a polar tube has penetrated (Schottelius et al. 2000). Similar events have been observed by using Encephalitozoon hellem in bovine pulmonary fibroblasts: polar tubes discharged its contents in an invagination of the host cell membrane (Bigliardi and Sacchi 2001). At the end of the invasion process the sporoplasms, that were previously forced through the polar tube, were occupied by a vacuole. Another published micrograph showed an Encephalitozoon intestinalis polar tube invaginating the plasma membrane of a macrophage (Magaud et al. 1997). Another scheme for the entry of microsporidia (E. intestinalis) into nonprofessional phagocytes (Caco-2 cells) involving specific interaction between the posterior pole of the microsporidian spore and the cellular membrane, extrusion of the polar tube, and induced phagocytosis of the sporoplasm within a host cell membrane-derived vacuole has been suggested (Foucault and Drancourt 2000). By contrast, other studies suggest that the phagocytic process occurs on contact of the spore apex with the host cell membrane (Magaud et al. 1997). However, no evidence for receptor-mediated endocytosis exists. So polar tube extension may be also an adaptation of the nonmotile spore to interact with more or less distant target cells. However, most of these concepts (Fig. 2) are still 


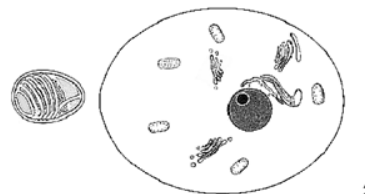

a

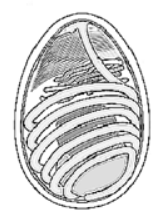

a
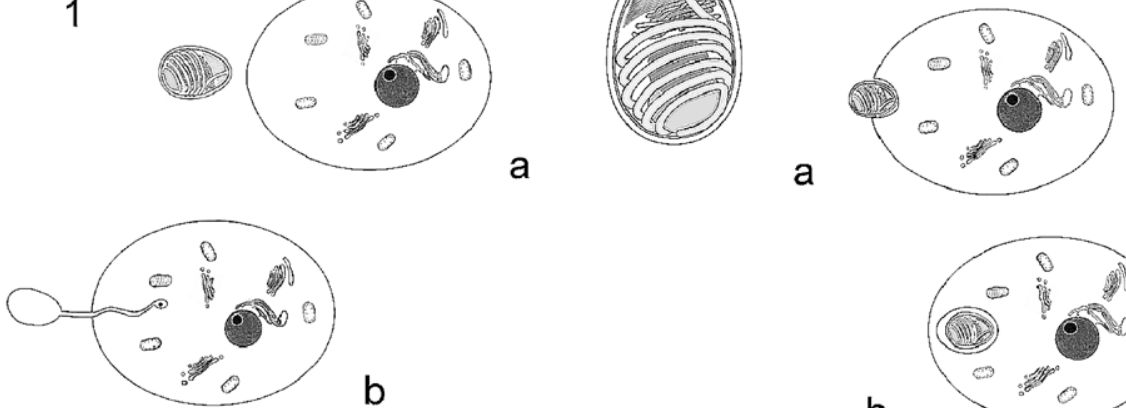

b

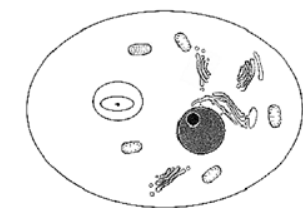

C

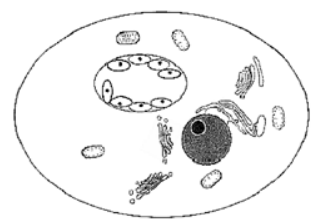

d

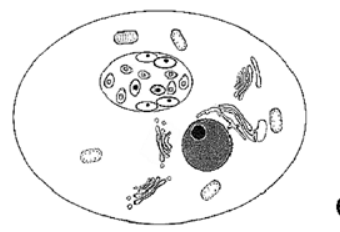

e

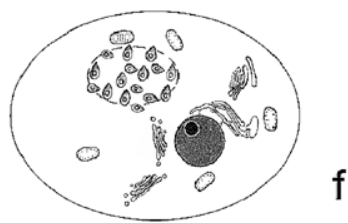

$f$

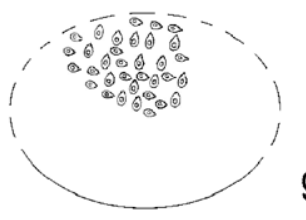

g b

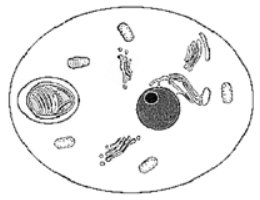

C

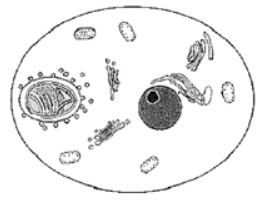

d
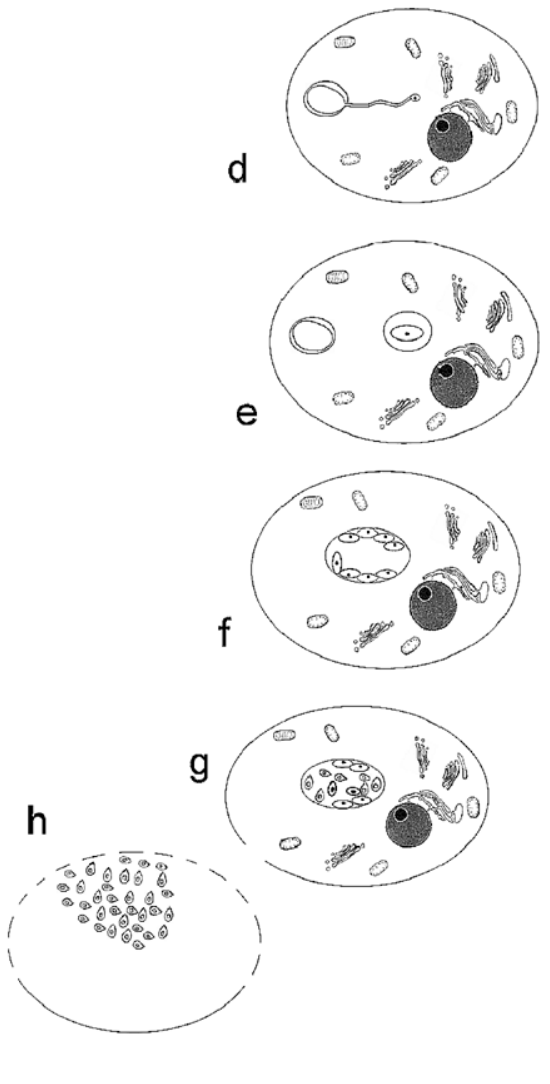

Fig. 3. There are two ways how microsporidia invade cells for which experimental evidence exists. 1. With appropriate conditions the polar tube is discharged through the anterior end of the spore thereby penetrating a new host cell (1b) and inoculating the infective sporoplasm into the new host cell. Inside the host cells, the sporoplasms that are released from the spores mature into meronts that are surrounded by a parasitophorous vacuole (in the case of the Encephalitozoon spp.) (1c). Meronts reproduce by binary fission, and finally develop into sporonts which are characterized by the appearance of a dense surface coat (1d). Sporonts divide into sporoblasts which will finally develop to mature spores (sporogony) (1e). New spores are released from the parasitophorous vacuole (1f) and from the host cell $(1 \mathrm{~g})$. 2. There is a different way for the spore to enter the host cell: We have clear evidence that microsporidian spores gain access to new host cells by endocytosis as well. Spores are phagocytosed by the cell (2a) and the phagosome with the phagocytosed spores matures to lysosomes (2c). Sporoplasms are able to escape from the maturing lysosomes and infect the cytoplasm of the cells by polar tube discharge (2d). Inside the host cells, the sporoplasms that are released from the spores mature into meronts and development will take place as described above (2e- $\mathrm{h})$. 
hypothetical and have to be verified because it is difficult to distinguish a polar tube discharging in an invagination of the host cell membrane from a cell engulfing a spore with previously extruded polar tube.

In addition to the infection of new host cells from outside (Fig. 3) there is now considerable evidence that phagocytosis of spores by several cells takes place as well (Couzinet et al. 2000, Franzen 2004, Franzen et al. 2005), but the intracellular fate of these phagocytosed microsporidian spores inside the cells have not been followed until recently. We have established a system using Encephalitozoon cuniculi as a model to investigate the cell invasion process and intracellular fate of microsporidia into host cells (Franzen et al. 2005). Most microsporidian spores enter the cytoplasm by internalisation of the spores without discharge of their polar tubes. This uptake could be inhibited by cytochalasin D and this inhibition suggests that the entry of spores into the cells is mediated by directed, actin-dependent phagocytosis. The phagosomes with the internalised microsporidian spores mature to endosomal and thereafter to lysosomal compartments, which has been shown by the colocalisation of internalised spores with several endosomal and lysosomal markers inside the cells (Franzen et al. 2005). In previous studies intracellular microsporidian spores in vacuoles showed a high capacity to block fusion with secondary lysosomes (Weidner 1975, Weidner and Sibley 1985), but it is important to differentiate between phagosomes harbouring internalised spores that will mature to lysosomes and parasitophorous vacuoles in which the development of the parasites inside the cells will take place. We have shown that the spores inside the lysosomal compartments are rapidly digested; however, some sporoplasms escape from the maturing lysosomes and infect the cytoplasm of the host cells by polar tube discharge (Fig. 3). The sporoplasms of these spores and of the spores that inject the polar tube from outside the cells seem to be the only source for the establishment of a new life cycle of the microsporidia inside the cells. These newly developing parasites were located inside vacuoles as well (in the case of the Encephalitozoon spp.), but these new compartments did not show any markers of late endosomal or lysosomal differentiation. The origin of this parasitophorous vacuole is still unclear.

\section{ORIGIN OF THE PARASITOPHOROUS VACUOLE}

Most microsporidian species develop in direct contact with the host cell cytoplasm and not within a vacuole but some microsporidia (e.g. Encephalitozoon spp. and Glugoides intestinalis) induce the formation of a surrounding membrane, presumably of host cell origin, known as the parasitophorous vacuole, at an early stage of infection. The origin of this parasitophorous vacuole is not yet fully understood. Ultrastructural examinations show that early developmental stages of Encephalitozoon spp. are surrounded by a tightly adhering membrane, and it has been suggested that the parasitopho- rous vacuole originates from the invaginated cell membrane after discharge of the tube into an invagination of the cell membrane (Magaud et al. 1997, Foucault and Drancourt 2000). This concept would be in agreement with the finding that the polar tube enters new host cells by a phagocytic process (see above). However, this proposal implies that the mode of cell invasion of the Encephalitozoon spp. into host cells is completely different from that of the other microsporidia. Although the diversity and abundance of the microsporidia is extensive, it seems not very likely that the unique extrusion apparatus of the microsporidia is used in totally different ways by the species that develop inside a parasitophorous vacuole and by those that develop in direct contact with the host cell cytoplasm. Moreover, it is difficult to distinguish between a polar tube extruding into a phagocytic cell invagination from an extruded polar tube that has been secondarily phagocytosed by a cell from a phagocytosed spore which has been secondarily extruded its polar tube. Therefore verification of this process is required utilizing microsporidian species developing in a parasitophorous vacuole in comparison with microsporidian species developing directly in the host cell cytoplasm.

\section{CONCLUSION}

Gaining access to cells by endocytosis and escaping destruction in the phago-/endo-/lysosome by egressing quickly from the phagocytic vacuole to multiply outside the lysosome is a common phenomenon in biology that has evolved several times during evolution. How this is put into execution by the microsporidia is a unique principle by which an obligate intracellular organism has managed this problem. The consequences for the efficiency of microsporidia to establish an intracellular infection have not yet been determined so far. If spore uptake results in efficient degradation of spores, phagocytosis might represent a defence mechanism that reduces the number of spores able to infect host cells by germination. Phagocytosis, however might not lead to the killing and digestion of all spores, so that germination of phagocytosed spores will occur and the parasites will escape from the phagolysosome by polar tube discharge. In this case, phagocytic uptake might add to the infectivity of microsporidia.

The unique extrusion apparatus of the microsporidia has obviously ensured the success of this phylum during evolution, resulting in a group of obligate intracellular organisms, capable of infecting almost any type of host and cell. It is not known how the polar tube penetrates host cell membranes or whether the polar tube or the spore binds to specific receptors on the host cell membranes. Additional characterisation of host cell attachment and penetration and of the intracellular fate of the parasites that lead to formation of the parasitophorous vacuole is imperative. This could lead to novel strategies for control of these important parasites. 
Acknowledgement. This work was supported by the German Bundesministerium für Bildung und Forschung [BMBF] -
Programm zur Förderung von Klinischen Forschergruppen in der Klinischen Infektiologie, Förderkennzeichen 01 KI 9952.

\section{REFERENCES}

BIGLIARDI E., SACCHI L. 2001: Cell biology and invasion of the microsporidia. Microbes Infect. 3: 373-379.

CALI A., TAKVORIAN P.M. 1999: Developmental morphology and life cycles of the microsporidia. In: M. Wittner and L.M. Weiss (Eds.), The Microsporidia and Microsporidiosis. AMS Press, Washington, D.C., pp. 85-128.

CALI A., TAKVORIAN P.M., WEISS L.M. 2002: Brachiola algerae spore membrane systems, their activity during extrusion, and a new structural entity, the multilayered interlaced network, associated with the polar tube and the sporoplasm. J. Eukaryot. Microbiol. 49: 164-174.

COUZINET S., CEJAS E., SCHITTNY J., DEPLAZES P., WEBER R., ZIMMERLI S. 2000: Phagocytic uptake of Encephalitozoon cuniculi by nonprofessional phagocytes. Infect. Immun. 68: 6939-6945.

FOUCAULT C., DRANCOURT M. 2000: Actin mediates Encephalitozoon intestinalis entry into the human enterocyte-like cell line, Caco-2. Microb. Pathog. 28: 51-58.

FRANZEN C. 2004: Microsporidia: how can they invade other cells? Trends Parasitol. 20: 275-279.

FRANZEN C., MÜLLER A., HARTMANN P., SALZBERGER B. 2005: Cell invasion and intracellular fate of Encephalitozoon cuniculi (Microsporidia). Parasitology 130: 285-292.

KEOHANE E.M., WEISS L.M. 1999: The structure, function, and composition of the microsporidian polar tube. In: M. Wittner and L.M. Weiss (Eds.), The Microsporidia and Microsporidiosis. AMS Press, Washington, D.C., pp. 196224.

MAGAUD A., ACHBAROU A., DESPORTES-LIVAGE I. 1997: Cell invasion by the microsporidium Encephalitozoon intestinalis. J. Eukaryot. Microbiol. 44: 81S.
SCHOTTELIUS J., SCHMETZ C., KOCK N.P., SCHULER T., SOBOTTKA I., FLEISCHER B. 2000: Presentation by scanning electron microscopy of the life cycle of microsporidia of the genus Encephalitozoon. Microbes Infect. 2: 1401-1406.

THÉLOHAN P. 1892: Observations sur les Myxosporidies et essai de classification de ces organismes. Bull. Soc. Philom. 4: 165-172.

THÉLOHAN P. 1894: Sur la présence d'une capsule à filament dans les spores des microsporidies. C. R. Acad. Sci. 118: 1425-1427.

VÁVRA J., LARSSON J.I.R. 1999: Structure of the Microsporidia. In: M. Wittner and L.M. Weiss (Eds.), The Microsporidia and Microsporidiosis. AMS Press, Washington, D.C., pp. 7-84.

VISVESVARA G.S. 2002: In vitro cultivation of microsporidia of clinical importance. Clin. Microbiol. Rev. 15: 401-413.

VIVARÈS C.P., MÉTÉNIER G. 2001: The microsporidian Encephalitozoon. Bioessays 23: 194-202.

WEIDNER E. 1972: Ultrastructural study of microsporidian invasion into cells. Z. Parasitenkd. 40: 227-242.

WEIDNER E. 1975: Interactions between Encephalitozoon cuniculi and macrophages. Parasitophorous vacuole growth and the absence of lysosomal fusion. Z. Parasitenkd. 47: 1-9.

WEIDNER E., SIBLEY L.D. 1985: Phagocytized intracellular microsporidian blocks phagosome acidification and phagosome-lysosome fusion. J. Protozool. 32: 311-317.

Accepted 7 January 2005 